\title{
Investigation of mixing chamber for experimental FGD reactor
}

\author{
Jan Novosád ${ }^{1, a}$, Petra Dančová ${ }^{1}$ and Tomáš Vít ${ }^{1}$ \\ ${ }^{1}$ Department of Power Engineering Equipment, Faculty of Mechanical Engineering, Technical University of Liberec, \\ Studentska 2, 46117, Liberec, Czech Republic
}

\begin{abstract}
This article deals with numerical investigation of flow and mixing of air and sulphur dioxide $\mathrm{SO}_{2}$ in designated mixing chamber. The mixing chamber is a part of experimental laboratory reactor designed for simulating the flue gas desulfurization (FGD) process. Aim of this work is the numerical investigation of effect of different mixing chamber geometries to mixture composition, especially to mass fraction of sulphur dioxide. Using of similar concentration of sulphur dioxide in the experimental reactor as in the real process is necessary to be able to make additional research. Conclusion describes the effect of different geometries of mixing chamber to mixing. The aim of this work is to develop perfectly works mixing chamber, which will be manufactured and then implemented into experimental FGD reactor. The results will be validated by experiment after the mixing chamber will be manufactured.
\end{abstract}

\section{Introduction}

Coal-fired power plants in the Czech Republic are still a significant source of electricity. They produce a large amount of gases, which are harmful for the environment, especially $\mathrm{NO}_{\mathrm{x}}$ and $\mathrm{SO}_{2}$. Amount of pollutants generated during combustion is several times more than the amount prescribed by legislation. To reduce the amount of $\mathrm{SO}_{2}$ in the emissions, it is necessary to install the flue gas cleaning device.

The process of desulfurization is very complicated and depends on many parameters. There are lot of chemical reactions, thermal effects and finally the fluid flow and heat transfer. Marocco and Inzoli in work [1] described the mathematical model with all governing equations and source terms. They used them in numerical simulation of a pilot plant open-spray tower.

These towers for FGD are several tens of meters tall and few meters wide cylinders. Processes in spray tower occurring at very small size. Therefore if we want to make numerical simulation, the computational mesh should be very fine, so it consists of several million cells.

Currently there is no computational capacity to make simulation in short time with sufficient accuracy. Therefore it is appropriate to create a scale model of spray tower, where we can simulate the FGD process. This model could be used to verify numerical simulations. The advantages of this equipment is lower prize and huge opportunities for research, because the real spray tower is very needful equipment in flue gas cleaning and there are no possibilities to change regimes or equipment while the power plant is running.
Principles of FGD process is well-known. It is based on reactions of sulphur dioxide with limestone. Products of these reactions are especially gypsum and clean air. A lot of researches are interested in research which consists improving efficiency of FGD, utilization of waste products, savings of chemical agents etc.

The whole process was described by many authors, f.e. by Córdoba in work [3]. Novosád and Vít in work [4] presented the beginning of their research of FGD. They found that they need to make an experimental reactor to verify their numerical results. One of prerequisites for the proper function of experimental reactor is the preparation of flue gas.

Aim of this work is to investigate effect of mixing chamber to mixing $\mathrm{SO}_{2}$ and air and using the results to design the mixing chamber component.

\section{Experimental reactor}

The experimental reactor is designated as a scale model of FGD spray tower with some modifications that allows operation under different conditions (regimes) and changings of components.

Block scheme of the reactor is shown in figure 1 . In real reactors the source of flue gas is the combustion of coal. To have better control over the concentration the gas cylinder filled with sulphur dioxide is used as a source of flue gas. Gas in the cylinder is very concentrated (over 99.9\%). Therefore it is necessary to mix it with air to gain the concentration which is usual in industrial process. This concentration is around $5000 \mathrm{mg} \cdot \mathrm{m}^{-3}$ which equals to mass fraction $0.166 \%$.

\footnotetext{
${ }^{a}$ Corresponding author: jan.novosad@tul.cz
} 
In the reaction zone should be same conditions as in the real reactor. Reaction zone is cuboid with square cross section. Section area of the reaction zone is

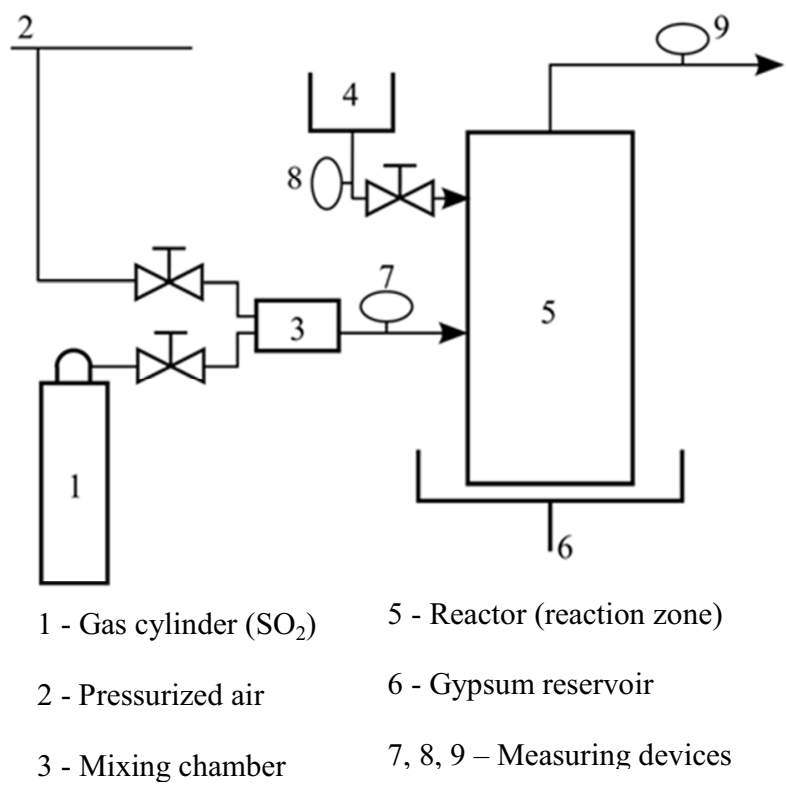

Figure 1. Scheme of experimental FGD reactor.

$\mathrm{S}=0.01 \mathrm{~m}^{2}$, vertical velocity of gases should be around $\mathrm{v}$ $=3 \mathrm{~m} \cdot \mathrm{s}^{-1}$. so the mass flux is:

$$
\dot{m}=\varrho \cdot S \cdot v,\left(\mathrm{~kg} \cdot \mathrm{s}^{-1}\right)
$$

where $\varrho$ is the density of mixture (air+SO2).

In our case:

$\dot{m}=1.2 \cdot 0.01 \cdot 3=0,0361\left(\mathrm{~kg} \cdot \mathrm{s}^{-1}\right)$

The mass flux of component $i$ is defined by:

$$
\dot{m}_{i}=w_{i} \cdot \dot{m} \quad\left(\mathrm{~kg} \cdot \mathrm{s}^{-1}\right)
$$

where $w_{i}$ is the mass fraction of $\mathrm{i}$-th component.

Following equation (3) using mass fractions described above leads to calculations of mass flux of air $\mathrm{m}_{\text {air }}=0.036 \mathrm{~kg} \cdot \mathrm{s}^{-1}$ and $\mathrm{SO}_{2} \mathrm{~m}_{\mathrm{SO} 2}=6 \cdot 10^{-5} \mathrm{~kg} \cdot \mathrm{s}^{-1}$. These fluxes are used as boundary conditions in numerical simulations.

\section{Numerical simulation}

Numerical investigation was performed in ANSYS Workbench environment, especially modules for creating models (Design Modeler), mesh (Meshing) was used. Ansys Fluent v. 16.0 was used for CFD calculations. Simulations were performed in three stages. In first stage the $2 \mathrm{D}$ mesh quality (element size) impact to results was tested. In the second stage different geometries were calculated using 2D mesh with element size determined in stage one. The last stage include simulations of the better variant evaluated from stage two using 3D mesh.

\subsection{Geometry of models}

The principle of mixing chamber is based on two inlets for each mixture component and one common outlet. For the investigation several types and variants of geometries were designed (see Figure 2). All geometries have similar dimensions of inlets, outlet, length of the chamber and distance between air and $\mathrm{SO}_{2}$ inlets.

The first type (see Fig. 2a) have the air inlet in the direction of main cavity, inlet of sulphur dioxide is connected from the side. The effect of the angle $\alpha$ of SO2 inlet tube was investigated. The angle varies in interval of $\alpha=\left\{15^{\circ}, 30^{\circ}, 45^{\circ}, 60^{\circ}, 75^{\circ}, 90^{\circ}\right\}$. Special variant of this geometry is the tube (nozzle) inserted to the axis of main cavity (see Fig. 2b).

The second one have $\mathrm{SO}_{2}$ inlet in the main direction and two (symmetric) air inlets from sides (see Fig. 2c). The effect of the angle $\alpha$ of air inlet tubes was investigated. The angle varies in interval of $\alpha=\left\{15^{\circ}, 30^{\circ}, 45^{\circ}\right\}$.

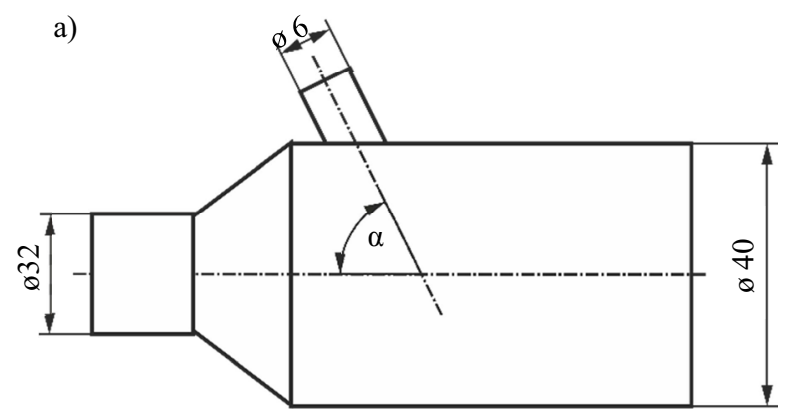

b)
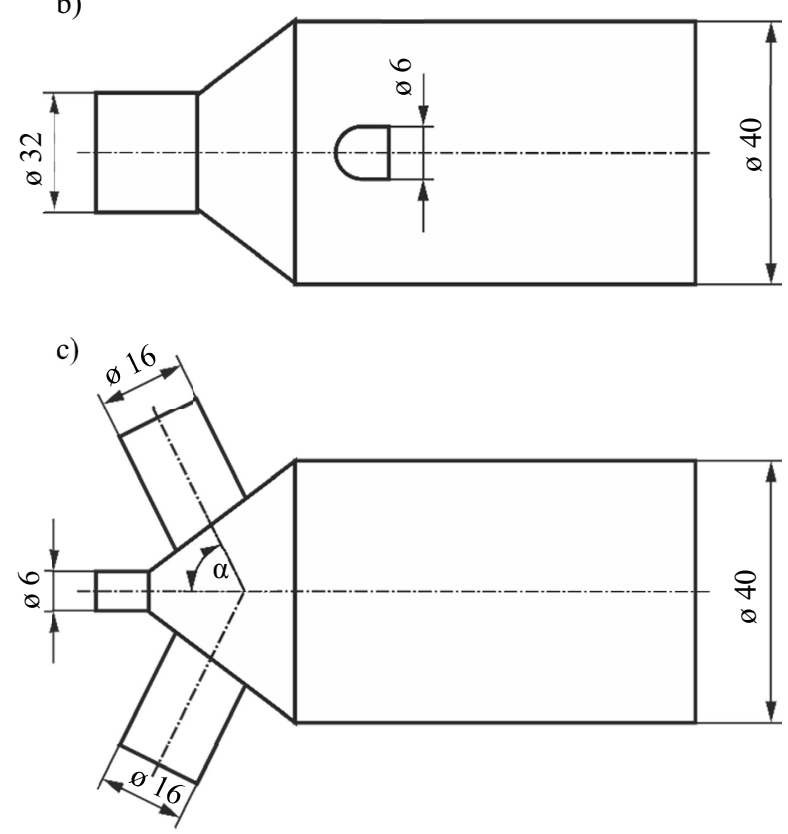

Figure 2. Sketches of model types and variants.

\subsection{Mesh}

Meshes for all models was created using ANSYS Meshing. 2D meshes consists of quad elements with different size varies of $\{0.2,0.5,0.7,1,2\}(\mathrm{mm})$. Labels of variants for $2 \mathrm{D}$ follow the scheme: „2D-el. size-angle_ $\alpha^{\prime \prime}$. Variant shown in figure 2b) is labelled as „2D-el. size-AX.“ Examples of meshes are shown in figure 3. 
After the evaluation of first stage of calculations, the suitable element size $(0.7 \mathrm{~mm})$ were determined and this element size was used for additional stages.

For 3D mesh hexahedral elements were used. Labels of variants for 3D follow the scheme: ,3D-angle_ $\alpha . “$

a) Coarse - el. size $2 \mathrm{~mm}$

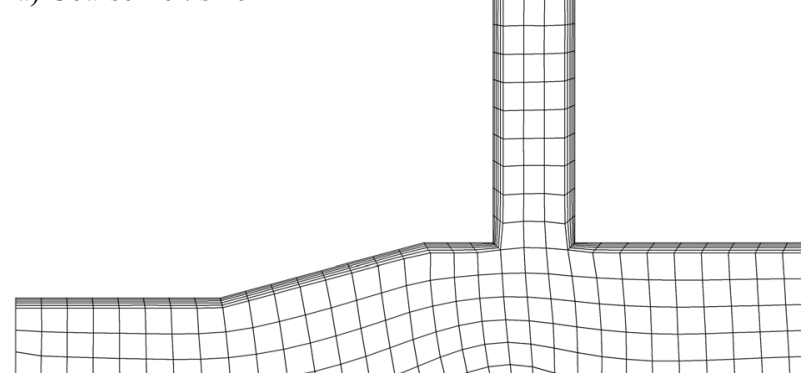

b) Medium - el. size $0.7 \mathrm{~mm}$

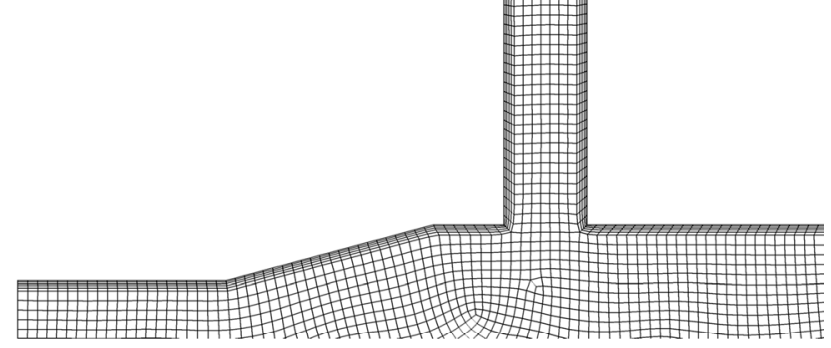

c) Fine - el. size $0.2 \mathrm{~mm}$

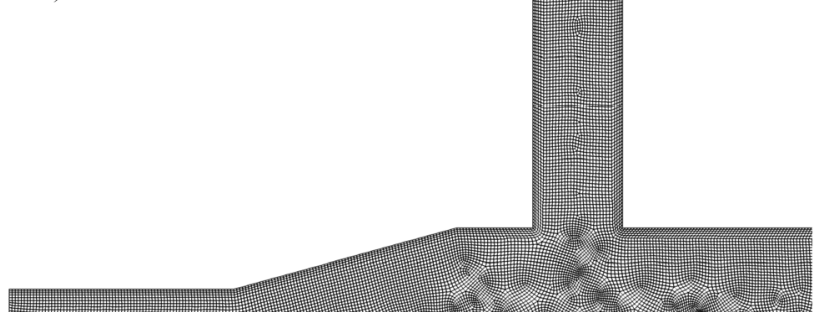

Figure 3. 2D meshes with different element sizes.

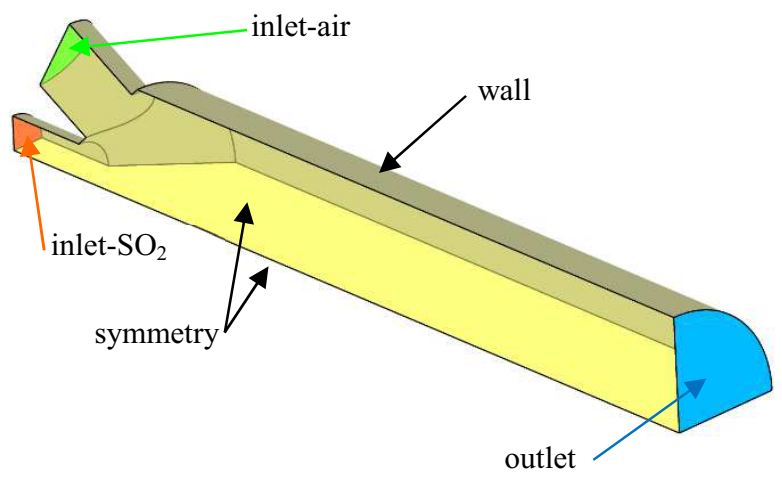

Figure 4. Boundary conditions - 3D model.

\subsection{Boundary conditions}

At the inlets of air and $\mathrm{SO}_{2}$ were assigned mass-flow-inlet boundary condition with mass flow value specified in section 2. Inlets of air are tubes with diameter of $32 \mathrm{~mm}$ (see Fig. 2a, b) or $2 \times 16 \mathrm{~mm}$ (see Fig. 2c). Inlets of $\mathrm{SO}_{2}$ are tubes with diameter of $6 \mathrm{~mm}$ in all cases.

At the outlet (tube with diameter of $40 \mathrm{~mm}$ ) were set pressure-outlet.

To simplify the 3D model symmetry boundary conditions were used. 3D model with labelled boundary conditions is shown in figure 4.

\subsection{Solution}

At the inlets of air and $\mathrm{SO}_{2}$ were assigned mass-flow-inlet boundary condition with mass flow value specified in section 2 .

Flue gas was considered as ideal gas mixture of air and $\mathrm{SO}_{2}$. The energy equation was set to on. Viscous model SST k- $\omega$ was used. We used steady flow and pressure based solver. Species transport model with no reactions was used.

\subsection{Methodology of the results assessment}

The results obtained by numerical simulation were mass fraction and velocity fields. Values at inlet and outlet boundary conditions were evaluated using Area-weighted-average method. To plot the mass fraction across the cross section, additional surfaces and lines were created.

To compare different geometries we use the mass fraction of $\mathrm{SO}_{2}$ to rate the desired function of mixing chamber, especially to gain desired mass fraction on outlet and its uniformly distribution in fluid flow.

Turbulent kinetic energy fields is displayed to show the efficiency of mixing and places where the mixing should be increasing by geometry modification.

Labels of variants for 2D follow the scheme of file names: „2D-el. size-angle“. For 3D the file names are in this way: ,3D-angle“.

\section{Results}

Results are divided into three sections accordingly to the stages of numerical simulations. Labels of variants for 2D follow the scheme of file names: „2D-el. size-angle“. For 3D the file names are in this way: ,3D-angle“"

\subsection{Mesh quality impact}

The mesh quality impact was investigated on $2 \mathrm{D}$ variant shown in figure 2a) with angle of $\mathrm{SO}_{2}$ inlet tube $\alpha=90^{\circ}$ and boundary conditions described in section 3.3 were used.

Profiles of mass fraction of $\mathrm{SO}_{2}$ across the outlet area is shown in figure 5 , detail of profile near the upper wall 


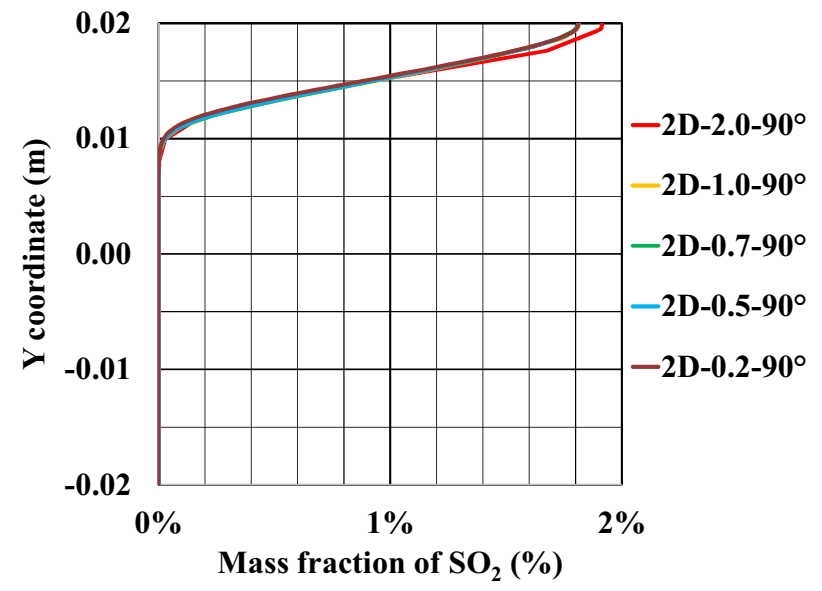

Figure 5. Mesh quality impact.

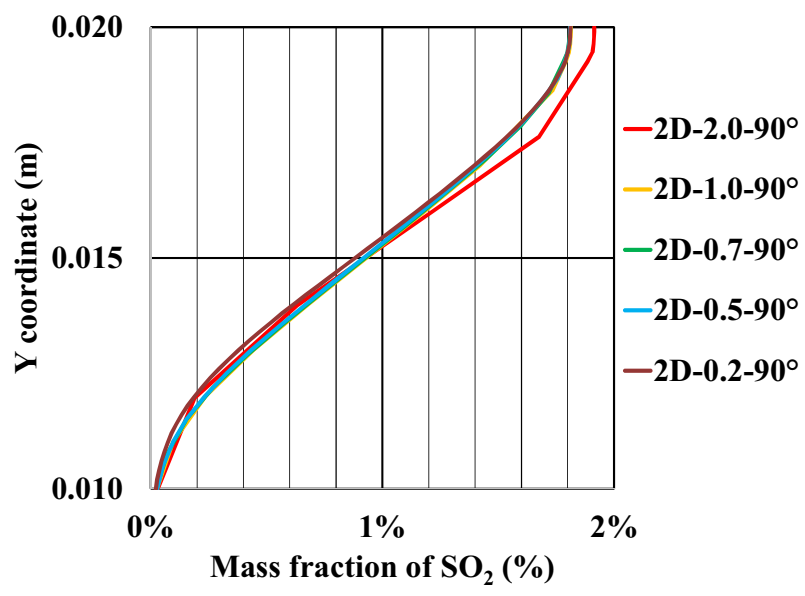

Figure 6. Mesh quality impact - near wall detail.

is shown in figure 6. Coordinate $\mathrm{Y}=0(\mathrm{~m})$ means the point on the tube axis.

It was founded that the coarse mesh (element size $0.2 \mathrm{~mm}$ ) causes higher mass fraction of $\mathrm{SO}_{2}$ than in other cases. Detailed graph (see Fig. 6) shows, that the curves for all cases have similar course. It can be said that curves for element size in the middle of the range are closer.

This leads to the conclusion that it is suitable to use mesh with element size in interval $\{0.5,1.0\}$ (mm). For additional cases element size $0.7 \mathrm{~mm}$ was chosen.

\subsection{Effect of $\mathrm{SO}_{2}$ tube angle}

The effect of the angle of $\mathrm{SO}_{2}$ inlet tube was investigated on $2 \mathrm{D}$ variant shown in figure $2 \mathrm{a}$ ) with angle of $\mathrm{SO}_{2}$ inlet tube varies in $\alpha=\left\{15^{\circ}, 30^{\circ}, 45^{\circ}, 60^{\circ}, 75^{\circ}, 90^{\circ}\right\}$. Additional simulated variant was the geometry shown in figure 2b). Mesh element size was set to $0.7 \mathrm{~mm}$ and boundary conditions described in section 3.3 were used.

Profiles of mass fraction of $\mathrm{SO}_{2}$ across the outlet area for different variants are shown in figure 7 . Coordinate $\mathrm{Y}=0(\mathrm{~m})$ means the point on the tube axis.

For individual variations it is evident that the mass fraction of $\mathrm{SO}_{2}$ depends insignificantly on the angle of $\mathrm{SO}_{2}$ inlet tube. To change the mass fraction profile across the outlet area, we need to change the geometrical arrangement (see Fig. 7, variant 2D-0.7-AX) to position the inlet of $\mathrm{SO}_{2}$ into the axis of main tube. Examples of spread of $\mathrm{SO}_{2}$ for two variants are shown in figure 8 using the $\mathrm{SO}_{2}$ enthalpy field, where the way of $\mathrm{SO}_{2}$ could be seen.

\subsection{Effect of air tube angle}

Following previous results the inlet of $\mathrm{SO}_{2}$ should be arranged into the axis of main tube. To provide simpler construction of mixing chamber the geometry concept follows figure 2c), where are two inlets of air on both sides of mixing zone. The effect of the angle of air inlets was investigated with angle of inlet tube varies in $\alpha=\left\{15^{\circ}, 30^{\circ}, 45^{\circ}\right\}$.

Velocity fields of $\mathrm{X}$-velocity (in the direction of main

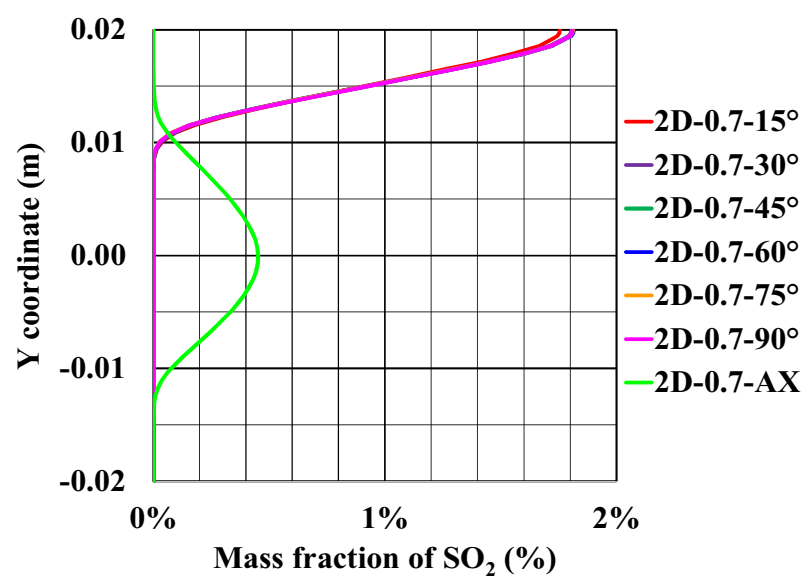

Figure 7. Effect of $\mathrm{SO}_{2}$ inlet tube angle.

a) $2 \mathrm{D}-0.7-45^{\circ}$

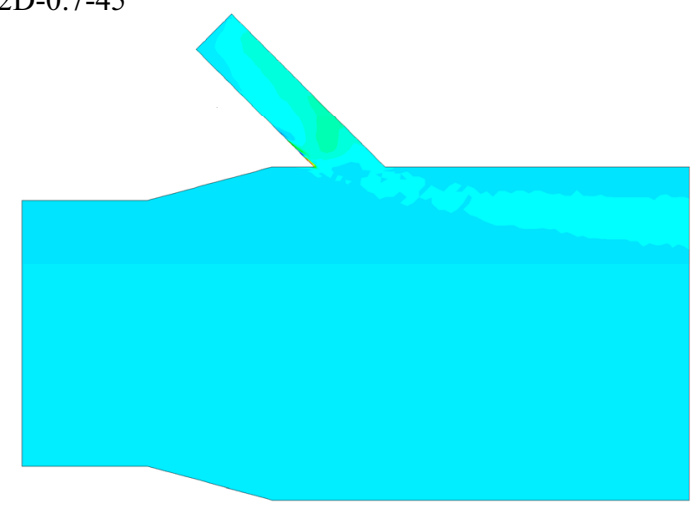

b) $2 \mathrm{D}-0.7-\mathrm{AX}$

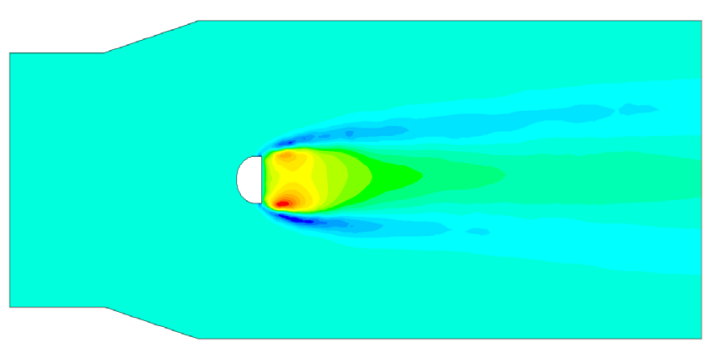

Figure 8. Spread of SO2 - examples. 
tube axis) for all cases on symmetry planes are shown in figure 9. This figure shows mirrored model, calculations were performed for the quarter of displayed model. In figure 9 can be observed that with increasing the angle of inlet air tube the zone with high velocity appears on the axis. With higher angle this zone is placed closer to $\mathrm{SO}_{2}$ inlet, which could cause the blockage in the sense of less amount of $\mathrm{SO}_{2}$, which can flow in the $\mathrm{X}$ direction, so it is deflected to the side of mixing chamber. This deflection causes more time spending in mixing chamber.

Profiles of mass fraction of $\mathrm{SO}_{2}$ in the outlet (see Figure 10) were evaluated in two directions of model [coordinate system - axis $\mathrm{Y}$ (vertical) and axis $\mathrm{Z}$ (horizontal)]. First figure for mass fraction in $\mathrm{Y}$ direction shows that the most uniform profile of mass fraction is for air inlet tube angle $\alpha=15^{\circ}$. In $Z$ direction is the situation exactly the opposite, the most uniform profile is for tube angle $\alpha=45^{\circ}$.

It can be assumed that the values of mass fraction are distributed between $\mathrm{Y}$ and $\mathrm{Z}$ axis. Therefore simply averaging of values in $\mathrm{Y}$ and $\mathrm{Z}$ direction was carried out. The result is shown in figure 11. From this figure we can see that the most uniform profile is for angle $\alpha=45^{\circ}$.

The best variant for application to design of mixing chamber could be illustrated by streamlines. Figure 12 shows streamlines begins at inlets of air (red lines) and $\mathrm{SO}_{2}$ (green lines). The figure illustrates higher mixing for the case with $\alpha=45^{\circ}$. In the zone where two fluid flows are interacting, the $\mathrm{SO}_{2}$ stream deflects and is brought into rotation which helps to mixing.

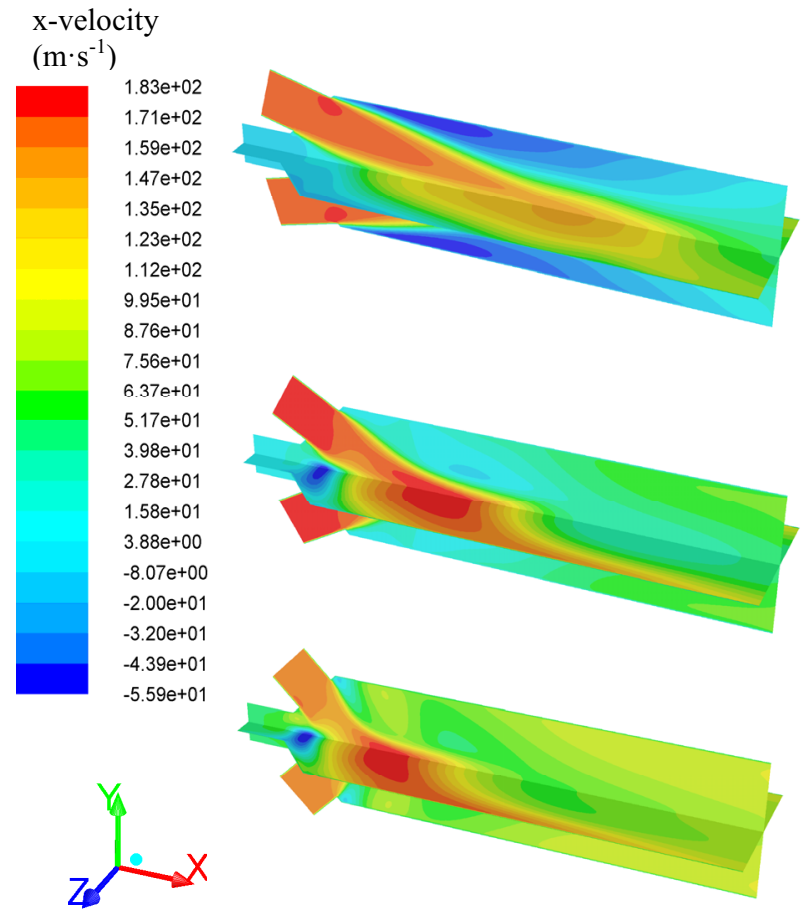

Figure 9. Contours of $\mathrm{x}$-velocity for $\alpha=15^{\circ}, 30^{\circ}, 45^{\circ}$. (from the top to bottom)
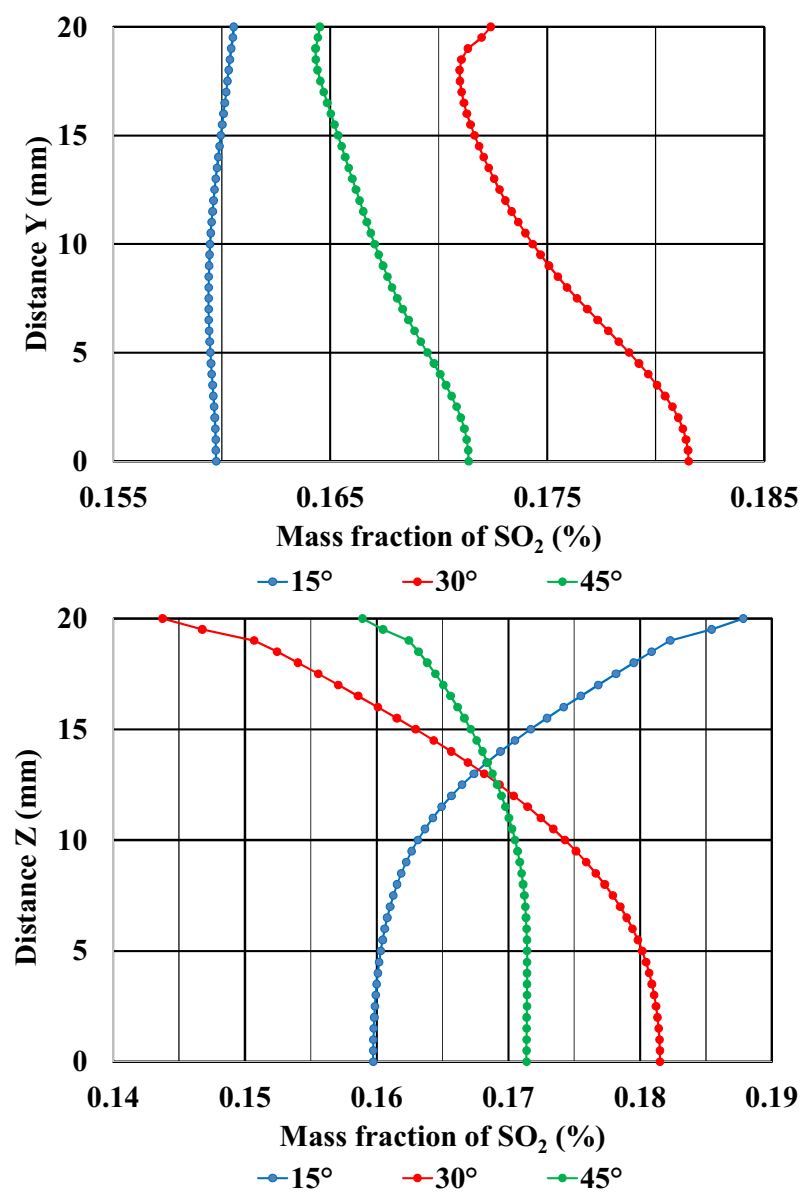

Figure 10. Mass fraction profile on outlet.

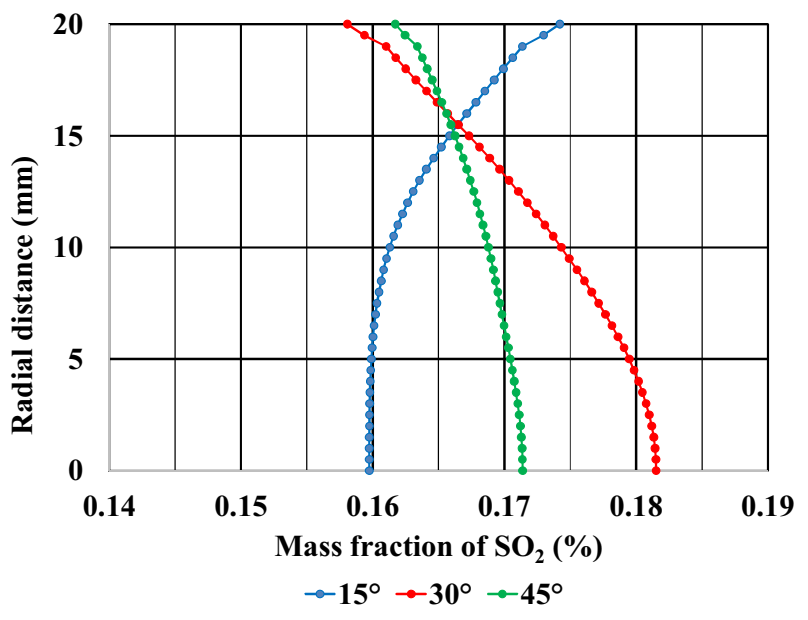

Figure 11. Averaged mass fraction profile on outlet. 


\section{Conclusion}

Numerical simulations performed in this work do not reflect the kinetics of chemical reactions. Simulations of various geometries of mixing chamber for mixing air and $\mathrm{SO}_{2}$ in three stages were performed. The calculations give detailed information about the distribution of the $\mathrm{SO}_{2}$ across mixing chamber outlet area. The results of each stage were used for next stage to gain the useful geometry for mixing chamber at the end of investigation.

During 2D simualtions for varies geometries was founded that the inlet of $\mathrm{SO}_{2}$ from the side of mixing chamber is not useful, because it causes high mass fraction of $\mathrm{SO}_{2}$ near the wall, but the zone around axis is not fullfilled. Better solution is to insert the $\mathrm{SO}_{2}$ inlet into the axis of mixing chamber.

In last stage $3 \mathrm{D}$ models of mixing chamber were simulated. The airflow was divided into two streams inputted by two inlets positioned on the sides of mixing chamber under angle $\alpha$. This embodiment is suitable for mixing because it allows uniform profile of $\mathrm{SO}_{2}$ concentration at the outlet. As the best option seems to be variant with air inlet tube angle $\alpha=45^{\circ}$. Maybe the additional research should be carried out to investigate the effect of more angles near to $45^{\circ}$.

The future work consider the construction design of whole mixing chamber including connection for hoses and pipes. After it will be manufactured the control measurement of $\mathrm{SO}_{2}$ fraction and velocity profiles will be performed. After that the mixing chamber becomes a part of experimental FGD reactor.

\section{Acknowledgement}

Author gratefully acknowledges financial support by MŠMT under the project CZ.1.07/2.2.00/28.0311 and project SGS 21000.

\section{References}

1. L. Marocco, F. Inzoli. Multiphase Euler-Lagrange CFD simulation applied to Wet Flue Gas Desulphurisation technology. International Journal of Multiphase Flow, 35, 2009.

2. Ansys Inc., Fluent User's Guide Release 16, 2015.

3. P. Córdoba. Status of Flue Gas Desulphurisation (FGD) systems from coal-fired power plants: Overview of the physic-chemical control processes of wet limestone FGDs. Fuel, Vol. 144, 2015.

4. J. Novosád, T. Vít. Numerical simulation of flow in the wet scrubber for desulfurization. Experimental Fluid Mechanics 2013, November 18.-21., 2014.

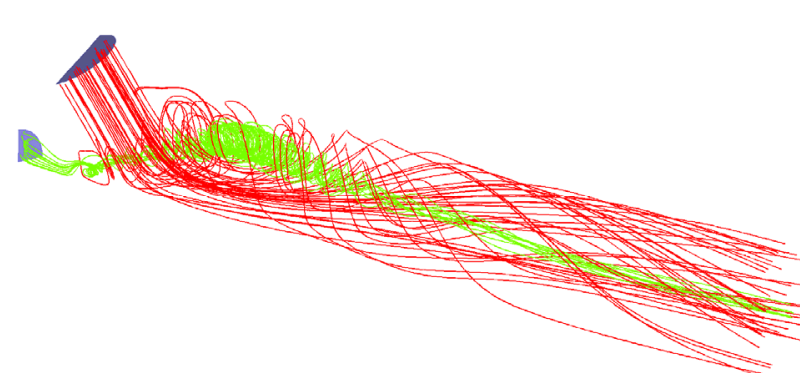

Figure 12. Streamlines of air and $\mathrm{SO}_{2}$ for $\alpha=45^{\circ}$. 